\title{
Relocalisation and Management for Smart System in WSN: A Review
}

\author{
Simran $^{1}$, Ms. Sonam Khattar ${ }^{2}$ \\ Computer Science \& Engineering Dept, Galaxy Global Group of Institutes, Kurukshetra ${ }^{1,2}$
}

\begin{abstract}
This work provides a review on relocalization of nodes in network in wireless sensor network. It provides a review on management of smart nodes network that will help to relocate it or by the use of controller. A routing protocol designed for WSN should have the ability of adapting to different applications and different network conditions it uses the concept of dynamic reconfiguration routing protocol that achieves the need of various applications and also various network conditions. As the result, it can be widely used in a military application for battlefield surveillance wireless sensor network has been an area of active research with many civilian application, fire detection, where it is difficult for humans to reach and once they are deployed, they work on their own and serve the data for which they are deployed In disaster control. The main objective of this work is to design mobility based network relocalization system in WSN. Localization is one of the most important applications for wireless sensor networks since the locations of the sensor nodes are critical to both network operations and most application level tasks.
\end{abstract}

Keywords: WSN System, Routing protocol, Dynamic Reconfiguration System, Localization, Mobility Management.

\section{INTRODUCTION}

With the expansion of micro-sensors and low-power wireless communications, the technology of WSN is becoming increasingly established step by step. An efficient routing service is a must require for all the applications running on the WSN platform. A lot of routing protocols specially designed for WSN have been proposed. The typical protocols include Gossiping Protocol, Sensor Protocols for information via Negotiation (SPIN), Directed-diffusion Routing, Greedy Perimeter Stateless Routing (GPSR), and Trajectory Based Routing (TBF). These routing protocols are all different from those protocols designed for the traditional. There are various applications running on the same WSN platform.

A wireless sensor network (WSN) is a network that is made of hundreds or thousands of these sensor nodes which are densely deployed in an unattended environment with the capabilities of sensing, wireless communications and computations (i.e., collecting and disseminating environmental data). wireless sensor network is the result of the combination of sensor techniques, embedded techniques, distributed information processing, and communication mechanisms. Many different routing, power management and data dissemination protocols have been designed for wireless sensor networks (WSNs), dependent on both the architecture of wireless sensor network (WSN) and the applications that WSN is intended to support.

Sensor nodes are collecting data about environment, after collecting it they process it and then transmit to the base station. Base station provides an interface between user and internet. Basic characteristic of the wireless sensor network are limited energy, dynamic network topology, lower power, node failure and mobility of the nodes, short-range broadcast communication and multi-hop routing and large scale of deployment. The benefit of structure wireless sensor network is that some nodes can be deployed with lower network maintenance and management cost. Fewer nodes can be deployed now since nodes are placed at specific locations to provide coverage while ad hoc deployment can have uncovered regions.

Most of the research in WSNs concerns networks whose nodes do not move and cannot be replaced. Nodes sense events of interest, and some energy efficient routing protocol is used for delivering the sensed data to static sinks. In this scenario it has been observed that the nodes that more than all the others have their energy drained from data communication are those closer to the sinks. These nodes relay data for all the other nodes in the network as well as packets from the sinks to the sensors. As a consequence, nodes that are closer to the sinks soon "die" from energy depletion resulting in the disconnection of the sinks from the rest of the network. We term the problem of energy drainage at the sink's neighbours the "sink's neighbours problem." The closer these nodes are to the sink, the higher the number of packets they receive and transmit, and consequently the higher their energy consumption.

The paper is ordered as follows. In section II, it represents related work of dynamic relocalisation system in WSN network. In Section III, It defines the dynamic reconfiguration scheme. The proposed system is defined in section IV. Finally, conclusion is explained in Section V. 
Vol. 6, Issue 4, April 2017

\section{RELATED WORK}

Jean A. Guevara et.al.[1] proposed a transducer electronic data sheet (TEDS) architecture for the management of reconfigurable WSNs nodes. To test the node architecture and validate its capability, the proposed TEDS was implemented for an environmental sensor network. Application of this proposed architecture to an environmental sensor network shows that the use of the new TEDS increases sensor nodes flexibility while its implementation does not affect the network performance in issues related to power consumption and bandwidth requirement.

Fei Ding et.al.[2] presented a hierarchical decentralized network reconfiguration approach to minimize power losses for smart distribution systems. It is shown that the decentralized approach can achieve similar results as other methods with significantly reduced computation time.The enhanced performance of the hierarchical decentralized approach is clearly established. the proposed hierarchical decentralized network reconfiguration method takes advantage of network decomposition and a multi-agent architecture to obtain the optimal configuration, and the computation time for obtaining the optimal configuration can be greatly reduced. Thus, the hierarchical decentralized approach is a promising option with reasonable tradeoffs between efficiency and accuracy in view of an increasing emphasis on implementing real-time distribution system automation.

Subhas Chandra M. et.al.[3] developed a Wearable Sensors for Human Activity Monitoring. They can be extremely useful in providing accurate and reliable information on people's activities and behaviours, thereby ensuring a safe and sound living environment. The human activity monitoring is a vibrant area of research and a lot of commercial development are reported. Wearable sensors in the form of panic buttons for emergency help have been in use for a long time and are a huge commercial success. Most importantly, the panic button should be light in weight so that it is comfortable to wear 24/7. The development of light-weight physiological sensors will lead to comfortable wearable devices to monitor different ranges of activities of inhabitants. Formal and Informal survey predicts an increase of interest and consequent usages of wearable devices in near future, the cost of the devices is also expected to fall resulting in of wide application in the society.

Bing Yuan et.al.[4] proposed the optimization research of node localization algorithm based on Wireless Sensor Network, focuses on DV-Hop localization algorithm, and gives its algorithm simulation and analysis of the simulation results. DV-Hop algorithm is one of the series algorithms which is put forward according to a distance vector routing algorithm and of GPS positioning principle. It can be seen that the improved algorithm improved positioning performance compared with the original algorithm, and improved point is from the actually situation. All improved points make the algorithm more suitable for practical application.

Abdul Waheed Khan et.al. [5] developed a VGDRA(A Virtual Grid based Dynamic Routes Adjustment) Scheme for mobile sink based Wireless Sensor Networks. VGDRA scheme aims to minimize the routes reconstruction cost of the sensor nodes while maintaining nearly optimal routes to the latest location of the mobile sink. VGDRA scheme partitions the sensor field into a virtual grid and constructs a virtual backbone structure comprised of the cell header nodes. A mobile sink while moving around the sensor field keeps on changing its location and interacts with the closest border-line cell-header for data collection. He also aim to develop a small test bed for the practical implementation of the proposed VGDRA scheme on real hardware (motes) and evaluate its results.

Mary M.,Vinay M.Pai et.al. [6] proposed a recent advances in wearable sensors for health monitoring and systems that monitor movement, physiology and environment, with a focus on applications for Parkinson's disease, stroke, and head and neck injuries. One of the key areas of opportunity is the development and optimization of techniques to measure other physiologic metrics with higher accuracy. A second key area of opportunity is the integration of environmental sensing and feedback to the wearer, enhancing sensory-feedback in the form of retinal and cochlear implants, and monitoring environmental risks such as radiation, pathogens, chemicals and poor air quality, and environmental parameters such as ambient temperature, light-levels, humidity, proximity and location. A third key area of opportunity is the development of automatic algorithms that can generate relevant clinical alerts based on changes in the physiological data.

Amir Ehsani et.al.[7] developed a Reliability-Oriented Single-Path Routing Protocols In Wireless Sensor Networks. He modelled the reliability of two different types of sensor nodes: energy harvesting sensor nodes (EHSNs) and batterypowered sensor nodes (BPSNs). He also presented wireless link failure models for each type of sensor nodes. In these models, we consider different parameters, such as battery life-time, shadowing, noise and location uncertainty on wireless link reliability.

Abhishek Majumder et.al.[8] proposed Design and Analysis of an Adaptive Mobility Management Scheme for Handling Internet Traffic in Wireless Mesh Network. Mesh networks with Mobility management and Mesh Mobility 
Management have been proposed. He proposed a session-to-mobility ratio (SMR) based mobility management scheme. The scheme enables the MC to send location update message to the gateway, uses forward chain, tunnelling and a threshold SMR value for reducing the cost of mobility management. Since mobility of the MC is considered for sending location update message to the GW, highly mobile MC will not send location update message frequently and location update cost will decrease.

Martin K. et.al.[9] proposed An Efficient Approach to Node Localisation and Tracking in Wireless Sensor Networks. Localisation is one of the most important applications for wireless sensor networks since the locations of the sensor nodes are critical to both network operations and most application level tasks. Numerous techniques for localisation of sensor nodes that make use of the Received Signal Strength Indicator (RSSI) have been proposed because of the simplicity and low cost of implementation. Development of a framework is to include a mathematical model of the antenna radiation pattern in a WSN node localisation algorithm. The model was developed for a 2-D plan.

Jong-H. Lee et.al.[10] developed Comparative Handover Performance Analysis of IPv6 Mobility Management Protocols. The existing IPv6 mobility management protocols developed by the IETF have been analysed and compared in terms of handover latency, handover blocking probability, and packet loss. From the conducted analysis results, the following are confirmed-Utilizing L2 information, employing buffering management, Wireless link condition, Network topology.

\section{ROUTING PROTOCOLS IN WSN}

\section{Location Based Protocols}

In location-based protocols, sensor nodes are simply addressed by their means of locations. In sensor networks, location information for nodes is necessary. To estimate energy consumption, all the routing protocols should calculate the distance between two particular nodes. We present commonly used location-aware routing protocols in WSNs.

\section{- $\quad$ Geographic Adaptive Fidelity (GAF)}

Geographic adaptive fidelity is an energy aware routing protocol. In GAF the sensor field will be separated into grid squares, each sensor uses its location information to associate with other grids. This location information will be provided by GPS or by other location systems.

\section{- Trajectory-Based Forwarding (TBF)}

TBF is a routing protocol that requires a sufficiently dense network and the presence of a coordinate system, for example, a GPS, so that the sensors can position themselves and estimate distance to their neighbours. The source specifies the trajectory in a packet, but does not explicitly indicate the path on a hop-by-hop basis. Based on the location information of its neighbours, a forwarding sensor makes a greedy decision to determine the next hop that is the closest to the trajectory fixed by the source sensor.

\section{- $\quad$ Geographic Random Forwarding (GeRaF)}

$\mathrm{GeRaF}$ assumes that all sensors are aware of their physical locations, as well as that of the sink. Although GeRaF integrates a geographical routing algorithm and an awake-sleep scheduling algorithm, the sensors are not required to keep track of the locations of their neighbors and their awake-sleep schedules. When a source sensor has sensed data to send to the sink, it first checks whether the channel is free in order to avoid collisions. If the channel remains idle for some period of time, the source sensor broadcasts a request-to-send (RTS) message to all of its active (or listening) neighbors. This message includes the location of the source and that of the sink.

\section{Mobility-based Protocols}

Mobility brings new challenges to routing protocols in WSNs. Sink mobility requires energy efficient protocols to guarantee data delivery originated from source sensors toward mobile sinks. In this section we discuss sample mobilitybased routing protocols for mobile WSNs.

\section{- $\quad$ Scalable Energy-Efficient Asynchronous Dissemination (SEAD)}

SEAD is self-organizing protocol, which was proposed to trade-off between minimizing the forwarding delay to a mobile sink and energy savings. SEAD considers data dissemination in which a source sensor reports its sensed data to multiple mobile sinks and consists of three main components namely dissemination tree (d-tree) construction, data dissemination, and maintaining linkages to mobile sinks. It assumes that the sensors are aware of their own geographic locations.

\section{- Dynamic Proxy Tree-Based Data Dissemination}

A dynamic proxy tree-based data dissemination framework was proposed for maintaining a tree connecting a source sensor to multiple sinks that are interested in the source. This helps the source disseminate its data directly to those mobile sinks. Because of target mobility, a source may change and a new sensor closer to the target may become a source. Each source is represented by a stationary source proxy and each sink is represented by a stationary sink proxy. 
Vol. 6, Issue 4, April 2017

\section{NEED AND DEVELOPMENT OF DYNAMIC RECONFIGURABLE SYSTEM IN WSN}

To design a WSN application, knowledge of many elements of the context is essential as they influence the operation greatly. During the development of such a WSN, it is unknown which influences the nodes might experience, which nodes might crash, and how long exactly the sensor nodes will last with the available energy.

Reconfiguration means adapting components or their arrangement within a system. Recently, techniques of dynamic reconfiguration have attracted increasing attention from the research community. These techniques enable reconfiguration of the sensor network hardware at run time to adapt to external dynamics, providing an innovative approach to designing an energy-efficient WSN in a highly dynamic environment. Due to advances in hardware technology, several reconfiguration techniques have been developed on the sensor node level. The utilization of all reconfiguration techniques have to consider dynamic factors, such as changes in user requirements, variations in communication channel quality, application changes, addition of new nodes, and node failure. This increases the complexity of using dynamic reconfiguration in WSNs. There are most commonly used techniques are given below.

\section{- $\quad$ Node Level Reconfiguration}

The dynamic reconfiguration at node level sought to minimize energy consumption by dynamically adjusting hardware platforms of sensor nodes. We addressed two promising reconfiguration hardware techniques, DVS and DMS, since they have already been separately used on computation and communication systems to reduce the energy consumption.

\section{- Dynamic Time Allocation}

When only limited time was available for the sensor node, it became critical to allocate the time resource for minimizing the total energy consumption. Such an allocation mechanism was called Dynamic Time Allocation (DTA), which determined the optimal share of computation time and transmission time subject to the time constraint.

\section{- $\quad$ Centralized Reconfiguration}

The need for reconfiguration of a WSN by creating a global model based on information coming from sensor nodes at run-time. Next, a design space search is performed in order to come up with a suitable new configuration, which is subsequently transferred on to the sensor nodes. Some provides a method for creating virtual machines for sensor nodes that execute small script-like programs. These scripts can be sent to a node and loaded and unloaded at run-time. This way, nodes can be reconfigured by uploading new individual software components to the nodes and deleting unused parts. Because in some cases this still requires a restart of individual nodes, it is not considered run-time adaptation.

\section{Node Localisation Parameters of WSN}

Localisation is one of the most important applications for wireless sensor networks since the locations of the sensor nodes are critical to both network operations and most application level tasks. Determining the location of nodes is one key application of Wireless Sensor Networks (WSN), for both civil and military applications. Most of the research efforts have focussed on the improvement of the localisation accuracy and complexity.

The first step for determining the location of a node is to find the distances between the respective nodes, which are assumed to be mobile, and some other nodes, which are assumed to be stationary. This is the so-called ranging phase. For unknown nodes' localization operation, estimate locations and the theory location of node will appear different results, this also called positioning error.

As shown in formula (4), each node positioning error is that use the location of the actual estimate and the real value of the node position to calculate as the follow equation:

$$
\text { error }=\frac{\sum_{\mathrm{i}=1}^{\mathrm{n}} \sqrt{(\text { xcal }-\mathrm{xreal})^{2}+(\text { ycal }- \text { yreal })^{2}}}{\mathrm{n}}
$$

The positioning error of the unknown node divided the ratio of the nodes in the wireless range is the positioning accuracy. In this the main consideration result is position error, the change of position precision and size to determine the positioning accuracy of improved algorithm and the original algorithm [6].

\section{Mobility Parameters of Sensor Nodes}

Mobility of Sensors Nodes Mobility is the major factor that affects the performance of the protocol. Due to high mobility of the nodes, unnecessary control information is exchanged and that can degrade the performance of entire network. Due to the excessive node movement in the network, it may be unstable and control overhead increases.

\section{PROPOSED SYSTEM}

In this paper, we proposed the WSN nodes have no fixed topology, but they can configure themselves to work in conditions such as the absence of permanent network infrastructure and in environments with limited or no human accessibility. Routing protocol designed for WSN should have the ability of adapting to different applications and different network conditions. To change a routing service in large scale sensor network propose a mobility based 
network reconfiguration system in WSN which can be dynamically reconfigured. It reviews the present mechanism of dynamic reconfiguration. Localization is one of the most important applications for wireless sensor networks since the locations of the sensor nodes are critical to both network operations and most application level tasks. The main parameter is the localization error of nodes. So, when all nodes get back to their location, there have some localization error but this value must be less for better response.

\section{CONCLUSION}

This work presents a review on network managaement system based on reconfiguration using controller. This work presents how to design a routing protocol, which can meet the need of different applications and different network conditions, is an extremely challenging problem. With the help of dynamic reconfiguration, the routing protocol can be changed by the remote administrator according to the need of different applications and different network conditions. In this work, all nodes are communicating with each other. A head is provided for giving the instructions to all nodes. The need for reconfiguration architecture for sensor network applications is apparent from the results of even a simple environmental monitoring algorithm. The main performance parameter will be localization error. With the help of this, it may prove the better stability of system. It takes the scenario of safety under wireless network. Due to this, it will provide safety and also minimum location error.

\section{REFFERENCES}

[1] Jean A. Guevara, Enrique A. Vargas,” Dynamically Reconfigurable WSN Node Based on ISO/IEC/IEEE 21451 TEDS”, IEEE Sensors Journal, Vol. 15, NO. 5, MAY 2015

[2] Fei Ding, and Kenneth A.” Hierarchical Decentralized Network Reconfiguration for Smart Distribution Systems-Part II: Applications to Test Systems", IEEE TRANSACTIONS ON POWER SYSTEMS, VOL. 30, NO. 2, MARCH 2015.

[3] Subhas Chandra Mukhopadhyay," Wearable Sensors for Human Activity Monitoring: A Review", IEEE SENSORS JOURNAL, VOL. 15, NO. 3, MARCH 2015.

[4] Bing Yuan,"The Optimization Research of Node Localization Algorithm Based on Wireless Sensor Network", 2014 7th International Conference on Bio Medical Engineering and Informatics (BMEI 2014).

[5] Abdul Waheed Khan,"VGDRA: A Virtual Grid based Dynamic Routes Adjustment Scheme for Mobile Sink based Wireless Sensor Networks", IEEE SENSORS JOURNAL， VOL. NO. 0, JANUARY 2014.

[6] Mary M. Rodgers, Vinay M. Pai, and Richard S. Conroy," Recent Advances in Wearable Sensors for Health Monitoring", DOI 10.1109/JSEN.2014.2357257, IEEE SENSOR JOURNAL,2014.

[7] Amir Ehsani Zonouz," Reliability-Oriented Single-Path Routing Protocols in Wireless Sensor Networks", DOI 10.1109/JSEN.2014.2332296, IEEE SENSORS JOURNAL SENSORS 2014.

[8] Abhishek Majumder,'Design and Analysis of an Adaptive Mobility Management Scheme for Handling Internet Traffic in Wireless Mesh Network", International Conference on Microelectronics, Communication and Renewable Energy (ICMiCR-2013).

[9] Martin K.,"An Efficient Approach to Node Localisation and Tracking in Wireless Sensor Networks",Globecom 2014.

[10] Jong-H.Lee ,"Comparative Handover Performance Analysis of IPv6 Mobility Management Protocols", IEEE TRANSACTIONS ON INDUSTRIAL ELECTRONICS, VOL. 60, NO. 3, MARCH 2013.

[11] Marcin Szczodrak," Dynamic Reconfiguration of Wireless Sensor Networks to Support Heterogeneous Applications", IEEE International Conference on Distributed Computing in Sensor Systems 2013.

[12] A. Di Marco," Implementing Adaptation and Reconfiguration Strategies in Heterogeneous WSN", IEEE 27th International Conference on Advanced Information Networking and Applications 2013.

[13] Reed Tompkins," Reconfiguration and Management in Wireless Sensor Networks", IEEE 2011.

[14] Sudip Misra," Detection of Identity-Based Attacks in Wireless Sensor Networks Using Signal prints", 2010 IEEE/ACM International Conference on Green Computing and Communications \& 2010 IEEE/ACM International Conference on Cyber, Physical and Social Computing, 2010.

[15] Anna A.," A case study for evaluating IEEE 802.15.4 wireless sensor network formation with mobile sinks", IEEE Communications Society subject matter experts for publication in the IEEE ICC 2009 proceedings. 